$$
\mathrm{H}_{2} \mathrm{~L}=\left[\mathrm{C}_{15} \mathrm{H}_{17} \mathrm{~N}_{5} \mathrm{O}\right]
$$

Table so. Crystal Data, Experimental Details of the X-ray Diffraction study and structure solution.

(a) Crystal Parameters at $293 \mathrm{~K}$

from a least-squares fit of the setting angles of 25 reflections with $9.0^{\circ}<\theta<13.0^{\circ}$

Chem. Formula $\quad \mathrm{C}_{15} \mathrm{H}_{17} \mathrm{~N}_{5} \mathrm{O}$
$\mathrm{FW}=283.34$
$\lambda=0.71073 \AA$
Crystal Habit:
yellow-brown plate
Crystal Dimensions:
Crystal System: Monoclinic
Space Group: $\mathrm{P} 2{ }_{1} / \mathrm{n}$ (No. 14)
$V=1513.0(3) \AA^{3}$
$\mathrm{a}=21.080(2) \AA$
$Z=4$
$\mathrm{b}=7.2369(9) \AA$
$F(000)=600$
$C=9.9220(11) \AA$
pcalc $=1.244 \mathrm{~g} \cdot \mathrm{cm}^{-3}$
$\beta=91.695(9)^{\circ}$
$\mu=0.083 \mathrm{~mm}^{-1}$

$0.50 \times 0.20 \times 0.10 \mathrm{~mm}$

Absences: hol: $h+1=2 \mathrm{~h}+1$

$0 \mathrm{k} 0: \mathrm{k}=2 \mathrm{n}+1$

(b) Measurement and treatment of Intensity Data

Instrument: Enraf-Nonius CAD4

Radiation: $\operatorname{MoK} \alpha(\lambda=0.71073 \AA)$ graphite monochromated

Detector window:

Height: $4.0 \mathrm{~mm}$

Width: $\quad 4.0 \mathrm{~mm}$

Scan mode: $\omega-2 \theta$

Scan width: $(0.80+0.35 \tan \theta)^{\circ}$
Scan speed: $0.91-8.24 \% / \mathrm{min}$

$\operatorname{Max} 2 \theta: 54^{\circ}$

$$
\left(\begin{array}{llllll}
h & -26,26 & k & 0,9 & 1 & 0,12
\end{array}\right)
$$

Standards :

Intensity: 3 refl. every $2 \mathrm{~h}$

Orientation: 3 refl. every 400 data refl.

Take-off angle: $2.95^{\circ}$

No. of refl. collected: 3482 


$$
\left[\mathrm{C}_{15} \mathrm{H}_{17} \mathrm{~N}_{5} \mathrm{O}\right]
$$

Page 2

Table so. (Continued)

(c) Treatment of Intensity Data

Reduction to $\mathrm{FO}^{2}$ and $\sigma\left(\mathrm{FO}^{2}\right)$, corrections for backgrounds, attenuator and LP in the usual manner [1]. No significative standard intensities variations $( \pm 0.5 \%)$. No absorption corrections. 3303 unique reflections $[$ Rint $=0.0274]$. No. of observed data $[I>2 \sigma(I)]: 1139$

(d) Structure Solution and Refinement

Direct methods using SHELXS-97 [2]. Full-matrix least-squares refinement using SHELXL-97 [3]. All non-H atoms anisotropic. N(3), C(8), C(9), C(10) and C(11) atoms were found disordered. Their occupancy factors were first refined, then kept fixed in the ratio 55/45. $\mathrm{H}$ atoms were introduced in calculations with the riding model, with Uiso equal to 1.1 times that of atom of attachment. Scattering factors were taken from "International Tables for Crystallography" [4]. Refinement on $\mathrm{FO}^{2}$ for all reflections. Weighted R-factors wR and goodnesses of fit $\mathrm{S}$ are based on $\mathrm{Fo}^{2}$, conventional R-factors $\mathrm{R}$ are based on Fo, with Fo set to zero for negative Fo ${ }^{2}$. Last refinement cycle: $\mathrm{wR}(\mathrm{all})=0.0701, \mathrm{R}(\mathrm{all})=0.0694, \mathrm{wR}(\mathrm{obs})=0.0657, \mathrm{R}(\mathrm{obs})=$ 0.0320 with 1139 obs. reflections > 46(Fo), 235 variable parameters, 
Table so. (Continued)

$\mathrm{w}=\left[\sigma^{2}\left(\mathrm{FO}^{2}\right)+(0.0317 \mathrm{P})^{2}\right]^{-1}$ where $\mathrm{P}=\left(\mathrm{FO}^{2}+2 \mathrm{FC}^{2}\right) / 3, \mathrm{~S}=0.578$,

$(\Delta / \sigma) \max =0.000,(\Delta / \sigma)$ mean $=0.000$, max. and min. residual peaks of

0.109 and $-0.116 \mathrm{e} / \AA^{3}$.

[1] Fair, C.K. Molen. Structure Solution Procedures, Enraf-Nonius, Delft, Holland, 1990.

[2] Sheldrick, G.M. SHELXS-97. Program for Crystal Structure Solution. Univ. of Göttingen: Göttingen, Germany, 1990.

[3] Sheldrick, G.M. SHELXL-97. Program for the refinement of crystal structures from diffraction data. Univ. of Göttingen: Göttingen, Germany, 1997.

[4] "International Tables for Crystallography", Vol. C, Kluwer Academic Publishers, Dordrecht, The Netherlands, 1992, Tables 4.2 .6 .8 and 6.1.1.4. 
Table s1. Atomic coordinates and equivalent isotropic displacement parameters $\left(\AA^{2} \times 100\right)$.

\begin{tabular}{|c|c|c|c|c|}
\hline Atom & $\mathrm{x} / \mathrm{a}$ & $y / b$ & $\mathrm{z} / \mathrm{C}$ & $\mathrm{Ueq}^{*}$ \\
\hline$O(1)$ & $0.52803(5)$ & $0.2729(2)$ & $0.2879(1)$ & $7.94(4)$ \\
\hline$N(1)$ & $0.56852(5)$ & $0.5694(2)$ & $0.4176(1)$ & $4.23(3)$ \\
\hline $\mathrm{N}(2)$ & $0.66660(6)$ & $0.3891(2)$ & $0.5641(1)$ & $6.29(4)$ \\
\hline $\mathrm{N}(4)$ & $0.59984(7)$ & $1.0179(2)$ & $0.5304(2)$ & $8.35(6)$ \\
\hline$N(5)$ & $0.73505(6)$ & $0.7452(2)$ & $0.7310(2)$ & $7.83(5)$ \\
\hline$C(1)$ & $0.48471(7)$ & $0.3830(2)$ & $0.2244(2)$ & $5.24(5)$ \\
\hline$C(2)$ & $0.44453(8)$ & $0.3036(2)$ & $0.1310(2)$ & $6.87(6)$ \\
\hline$C(3)$ & $0.39806(8)$ & $0.4098(3)$ & $0.0621(2)$ & $6.80(6)$ \\
\hline$C(4)$ & $0.39611(8)$ & $0.5991(2)$ & $0.0920(2)$ & $7.47(6)$ \\
\hline$C(5)$ & $0.43686(7)$ & $0.6745(2)$ & $0.1854(2)$ & $6.76(6)$ \\
\hline$C(6)$ & $0.48273(7)$ & $0.5709(2)$ & $0.2558(2)$ & $4.33(4)$ \\
\hline$C(7)$ & $0.52408(7)$ & $0.6573(2)$ & $0.3523(2)$ & $4.73(4)$ \\
\hline $\mathrm{N}(3)$ & $0.3485(3)$ & $0.3247(12)$ & $-0.0115(7)$ & $6.7(2) \neq$ \\
\hline$C(8)$ & $0.2917(3)$ & $0.4305(6)$ & $-0.0617(5)$ & $8.1(1) \neq$ \\
\hline C (9) & $0.3035(4)$ & $0.5254(9)$ & $-0.1852(6)$ & $14.3(3) \neq$ \\
\hline$C(10)$ & $0.3486(3)$ & $0.1252(7)$ & $-0.0322(7)$ & $5.9(2) \neq$ \\
\hline$C(11)$ & $0.3918(3)$ & $0.0357(6)$ & $-0.1305(5)$ & $9.4(2) \neq$ \\
\hline$N\left(3^{\prime}\right)$ & $0.3686(4)$ & $0.3444(14)$ & $-0.0556(9)$ & $7.7(3) \neq \neq$ \\
\hline$C\left(8^{\prime}\right)$ & $0.3288(3)$ & $0.4483(8)$ & $-0.1418(6)$ & $5.5(1) \neq \neq$ \\
\hline C ( $\left.9^{\prime}\right)$ & $0.2622(2)$ & $0.4962(8)$ & $-0.0996(7)$ & $7.0(2) \neq \neq$ \\
\hline$C\left(10^{\prime}\right)$ & $0.3780(4)$ & $0.1625(9)$ & $-0.1153(8)$ & $9.9(2) \neq \neq$ \\
\hline$C\left(11^{\prime}\right)$ & $0.3378(5)$ & $0.0447(12)$ & $-0.0404(11)$ & $13.3(4) \neq \neq$ \\
\hline C (12) & $0.60964(7)$ & $0.6651(2)$ & $0.5058(2)$ & $4.22(4)$ \\
\hline$C(13)$ & $0.65624(7)$ & $0.5732(2)$ & $0.5733(2)$ & $4.50(4)$ \\
\hline$C(14)$ & $0.60466(8)$ & $0.8619(2)$ & $0.5223(2)$ & $5.57(5)$ \\
\hline$C(15)$ & $0.69961(7)$ & $0.6710(2)$ & $0.6615(2)$ & $5.28(5)$ \\
\hline
\end{tabular}

Ueq $=1 / 3$ of the trace of the orthogonalized $U_{i j}$ tensor. キOccupancy factor $=0.55$. $\neq \neq$ Occupancy factor $=0.45$. 


$$
\left[\mathrm{C}_{15} \mathrm{H}_{17} \mathrm{~N}_{5} \mathrm{O}\right]
$$

Table s2. Hydrogen atomic positional and thermal parameters $\left(\AA^{2} \times 100\right)$.

\begin{tabular}{|c|c|c|c|c|}
\hline Atom & $\mathrm{x} / \mathrm{a}$ & $y / b$ & $\mathrm{z} / \mathrm{c}$ & $\mathrm{U}$ \\
\hline $\mathrm{H}(1)$ & 0.5132 & 0.2322 & 0.3574 & 8.7 \\
\hline $\mathrm{H}(2 \mathrm{~A})$ & 0.6425 & 0.3223 & 0.5124 & 6.9 \\
\hline $\mathrm{H}(2 \mathrm{~B})$ & 0.6973 & 0.3389 & 0.6101 & 6.9 \\
\hline $\mathrm{H}(2)$ & 0.4479 & 0.1780 & 0.1126 & 7.6 \\
\hline $\mathrm{H}(4)$ & 0.3665 & 0.6741 & 0.0472 & 8.2 \\
\hline $\mathrm{H}(5)$ & 0.4340 & 0.8004 & 0.2031 & 7.4 \\
\hline $\mathrm{H}(7)$ & 0.5189 & 0.7827 & 0.3692 & 5.2 \\
\hline $\mathrm{H}(8 \mathrm{~A})$ & 0.2564 & 0.3460 & -0.0759 & $8.9 \neq$ \\
\hline $\mathrm{H}(8 \mathrm{~B})$ & 0.2799 & 0.5195 & 0.0062 & $8.9 \ddagger$ \\
\hline $\mathrm{H}(9 \mathrm{~A})$ & 0.2663 & 0.5931 & -0.2134 & $15.7 \neq$ \\
\hline $\mathrm{H}(9 \mathrm{~B})$ & 0.3138 & 0.4373 & -0.2534 & $15.7 \neq$ \\
\hline $\mathrm{H}(9 \mathrm{C})$ & 0.3383 & 0.6095 & -0.1714 & $15.7 \neq$ \\
\hline $\mathrm{H}(10 \mathrm{~A})$ & 0.3056 & 0.0901 & -0.0585 & $6.4 \neq$ \\
\hline $\mathrm{H}(10 \mathrm{~B})$ & 0.3577 & 0.0689 & 0.0550 & $6.4 \neq$ \\
\hline $\mathrm{H}(11 \mathrm{~A})$ & 0.3844 & -0.0952 & -0.1319 & $10.3 \neq$ \\
\hline $\mathrm{H}(11 \mathrm{~B})$ & 0.4352 & 0.0594 & -0.1037 & $10.3 \neq$ \\
\hline $\mathrm{H}(11 \mathrm{C})$ & 0.3835 & 0.0857 & -0.2189 & $10.3 \neq$ \\
\hline $\mathrm{H}(8,1)$ & 0.3249 & 0.3822 & -0.2267 & $6.0 \neq \ddagger$ \\
\hline $\mathrm{H}(8,2)$ & 0.3504 & 0.5636 & -0.1599 & $6.0 \neq \neq$ \\
\hline $\mathrm{H}\left(9^{\prime} 1\right)$ & 0.2405 & 0.5622 & -0.1712 & $7.7 \neq \neq$ \\
\hline $\mathrm{H}(9,2)$ & 0.2645 & 0.5721 & -0.0203 & $7.7 \neq \neq$ \\
\hline $\mathrm{H}(9,3)$ & 0.2395 & 0.3847 & -0.0807 & $7.7 \ddagger \ddagger$ \\
\hline $\mathrm{H}(10 \mathrm{C})$ & 0.3656 & 0.1625 & -0.2102 & $10.9 \neq \neq$ \\
\hline $\mathrm{H}(10 \mathrm{D})$ & 0.4220 & 0.1243 & -0.1056 & $10.9 \neq \neq$ \\
\hline $\mathrm{H}(11 \mathrm{D})$ & 0.3433 & -0.0809 & -0.0689 & $14.6 \neq \neq$ \\
\hline $\mathrm{H}(11 \mathrm{E})$ & 0.2944 & 0.0808 & -0.0558 & $14.6 \neq \neq$ \\
\hline $\mathrm{H}(11 \mathrm{~F})$ & 0.3487 & 0.0550 & 0.0539 & $14.6 \neq \neq$ \\
\hline
\end{tabular}

‡Occupancy factor $=0.55 . \neq \neq$ Occupancy factor $=0.45$. 
Table s3. Final anisotropic displacement parameters $\left(\AA^{2} \times 100\right)$.

\begin{tabular}{|c|c|c|c|c|c|c|}
\hline Atom & U11 & $\mathrm{U} 22$ & U33 & $\mathrm{U} 12$ & U13 & U23 \\
\hline$O(1)$ & $8.29(9)$ & $5.03(7)$ & $10.2(1)$ & $-0.48(8)$ & $-3.92(8)$ & $1.70(7)$ \\
\hline $\mathrm{N}(1)$ & $3.75(7)$ & $3.87(7)$ & $5.00(8)$ & $0.04(7)$ & $-1.14(6)$ & $-0.16(6)$ \\
\hline $\mathrm{N}(2)$ & $6.16(9)$ & $4.37(8)$ & $8.1(1)$ & $-0.51(8)$ & $-3.26(8)$ & $0.51(7)$ \\
\hline$N(4)$ & $9.4(1)$ & $4.41(9)$ & $11.1(2)$ & $-1.0(1)$ & $-3.7(1)$ & $0.30(9)$ \\
\hline$N(5)$ & $7.6(1)$ & $6.6(1)$ & $9.0(1)$ & $-0.6(1)$ & $-3.49(9)$ & $-1.67(9)$ \\
\hline$C(1)$ & $4.8(1)$ & $4.05(9)$ & $6.7(1)$ & $0.12(9)$ & $-2.23(9)$ & $0.96(8)$ \\
\hline$C(2)$ & $6.9(1)$ & $4.9(1)$ & $8.6(1)$ & $-2.3(1)$ & $-3.2(1)$ & $0.95(9)$ \\
\hline$C(3)$ & $6.9(1)$ & $5.4(1)$ & $7.9(2)$ & $-1.9(1)$ & $-3.2(1)$ & $0.6(1)$ \\
\hline$C(4)$ & $8.4(1)$ & $4.4(1)$ & $9.3(2)$ & $-0.4(1)$ & $-4.9(1)$ & $0.8(1)$ \\
\hline$C(5)$ & $7.4(1)$ & $4.2(1)$ & $8.4(1)$ & $0.1(1)$ & $-3.8(1)$ & $-0.31(9)$ \\
\hline$C(6)$ & $4.21(9)$ & $3.95(9)$ & $4.8(1)$ & $0.35(8)$ & $-1.18(8)$ & $-0.37(7)$ \\
\hline$C(7)$ & $4.36(9)$ & $4.21(9)$ & $5.6(1)$ & $-0.21(9)$ & $-0.47(9)$ & $-0.09(8)$ \\
\hline $\mathrm{N}(3)$ & $6.0(4)$ & $6.4(3)$ & $7.4(4)$ & $-2.0(3)$ & $-3.7(3)$ & $0.8(3)$ \\
\hline$C(8)$ & $8.5(4)$ & $7.3(3)$ & $8.5(4)$ & $0.6(3)$ & $-1.8(4)$ & $-0.1(3)$ \\
\hline$C(9)$ & $17.7(8)$ & $14.0(6)$ & $11.0(5)$ & $0.9(4)$ & $-3.2(5)$ & $-4.4(5)$ \\
\hline$C(10)$ & $5.8(3)$ & $5.8(4)$ & $5.9(3)$ & $-1.2(3)$ & $-2.2(3)$ & $-1.6(3)$ \\
\hline$C(11)$ & $14.0(5)$ & $4.9(3)$ & $9.3(4)$ & $-1.4(3)$ & $1.6(3)$ & $0.2(3)$ \\
\hline $\mathrm{N}\left(3^{\prime}\right)$ & $7.0(5)$ & $6.0(4)$ & $9.8(6)$ & $-3.9(4)$ & $-2.6(4)$ & $0.7(3)$ \\
\hline$C\left(8^{\prime}\right)$ & $5.8(3)$ & $6.8(4)$ & $3.8(3)$ & $-1.1(3)$ & $-0.9(3)$ & $-0.4(3)$ \\
\hline$C\left(9^{\prime}\right)$ & $6.1(3)$ & $7.5(4)$ & $7.5(4)$ & $2.5(3)$ & $-1.0(3)$ & $-0.2(3)$ \\
\hline$C\left(10^{\prime}\right)$ & $9.1(5)$ & $13.8(7)$ & $6.8(4)$ & $-0.8(5)$ & $-0.9(4)$ & $-4.0(5)$ \\
\hline$C\left(11^{\prime}\right)$ & $12.5(7)$ & $10.2(8)$ & $17.3(9)$ & $3.1(7)$ & $1.6(6)$ & $2.4(6)$ \\
\hline$C(12)$ & $4.36(9)$ & $3.29(8)$ & $5.0(1)$ & $-0.22(8)$ & $-0.73(8)$ & $-0.56(7)$ \\
\hline$C(13)$ & $4.51(9)$ & $3.8(1)$ & $5.1(1)$ & $-0.17(9)$ & $-1.55(8)$ & $-0.78(8)$ \\
\hline$C(14)$ & $5.8(1)$ & $4.5(1)$ & $6.3(1)$ & $-0.7(1)$ & $-2.5(1)$ & $-0.13(9)$ \\
\hline$C(15)$ & $5.4(1)$ & $4.6(1)$ & $5.7(1)$ & $0.64(9)$ & $-1.27(9)$ & $-0.84(9)$ \\
\hline
\end{tabular}




$$
\left[\mathrm{C}_{15} \mathrm{H}_{17} \mathrm{~N}_{5} \mathrm{O}\right]
$$

Page 7

Table s4. Bond lengths ( $\AA$ ) and angles (deg) with e.s.d.'s in parentheses.

\section{Bond Lengths}

$O(1)-C(1)$
$C(1)-C(2)$
$C(2)-C(3)$
$C(3)-C(4)$
$C(3)-N(3)$
$N(3)-C(8)$
$N(3)-C(10)$
$C(8)-C(9)$
$C(10)-C(11)$
$C(7)-N(1)$
$C(12)-C(13)$
$C(12)-C(14)$
$C(14)-N(4)$

Bond Angles

$$
\begin{aligned}
& C(4)-C(5) \\
& C(5)-C(6) \\
& C(6)-C(1) \\
& C(6)-C(7) \\
& C(3)-N\left(3^{\prime}\right) \\
& N\left(3^{\prime}\right)-C\left(8^{\prime}\right) \\
& N\left(3^{\prime}\right)-C\left(10^{\prime}\right) \\
& C\left(8^{\prime}\right)-C\left(9^{\prime}\right) \\
& C\left(10^{\prime}\right)-C\left(11^{\prime}\right) \\
& N(1)-C(12) \\
& C(13)-N(2) \\
& C(13)-C(15) \\
& C(15)-N(5)
\end{aligned}
$$

1.3593 (19)

1.3946 (19)

$1.396(2)$

1.4207 (19)

$1.389(9)$

$1.400(10)$

$1.459(11)$

$1.516(8)$

$1.427(10)$

$1.3970(16)$

$1.3536(18)$

$1.434(2)$

$1.1368(17)$
$O(1)-C(1)-C(6)$
$119.60(14)$
$C(6)-C(1)-C(2)$
$120.65(16)$
$120.8(4)$
$120.9(4)$
$121.8(6)$
$120.3(6)$
$117.7(5)$
$111.9(8)$
$121.5(6)$
$\mathrm{N}(3)-\mathrm{C}(10)-\mathrm{C}(11)$
$120.95(16)$
$115.90(14)$
$123.85(14)$
$C(1)-C(6)-C(7)$
$\mathrm{C}(7)-\mathrm{N}(1)-\mathrm{C}(12)$
$119.75(13)$
$121.11(14)$
$119.10(15)$

$\mathrm{O}(1)-\mathrm{C}(1)-\mathrm{C}(2)$

$122.66(15)$

$C(2)-C(3)-C(4)$

$\mathrm{C}(2)-\mathrm{C}(3)-\mathrm{N}\left(3^{\prime}\right)$

$\mathrm{N}\left(3^{\prime}\right)-\mathrm{C}(3)-\mathrm{C}(4)$

$\mathrm{C}(3)-\mathrm{N}\left(3^{\prime}\right)-\mathrm{C}\left(8^{\prime}\right)$

$\mathrm{C}(3)-\mathrm{N}\left(3^{\prime}\right)-\mathrm{C}\left(10^{\prime}\right)$

$\mathrm{C}\left(8^{\prime}\right)-\mathrm{N}\left(3^{\prime}\right)-\mathrm{C}\left(10^{\prime}\right)$

$N\left(3^{\prime}\right)-C\left(8^{\prime}\right)-C\left(9^{\prime}\right)$

$\mathrm{N}\left(3^{\prime}\right)-\mathrm{C}\left(10^{\prime}\right)-\mathrm{C}\left(11^{\prime}\right)$

$C(4)-C(5)-C(6)$

$C(5)-C(6)-C(7)$

$\mathrm{C}(6)-\mathrm{C}(7)-\mathrm{N}(1)$

$119.85(13)$

$\mathrm{C}(12)-\mathrm{C}(13)-\mathrm{N}(2)$

$C(12)-C(13)-C(15)$

$\mathrm{N}(2)-\mathrm{C}(13)-\mathrm{C}(15)$

$\mathrm{C}(13)-\mathrm{C}(15)-\mathrm{N}(5)$

$117.74(15)$

$117.10(16)$

$120.7(4)$

$119.7(5)$

$125.1(8)$

$125.8(8)$

$108.8(7)$

$119.8(8)$

$103.7(9)$

$122.73(15)$

$120.25(15)$

$122.91(14)$

$124.67(14)$

$120.11(14)$

$115.21(14)$

$178.32(18)$

\section{Hydrogen Bonds}

\begin{tabular}{lllllll}
\hline D & H & D-H & A & H...A & D..A & D-H...A \\
\hline $\mathrm{N}(2)$ & $\mathrm{H}(2 \mathrm{~A})$ & 0.86 & $\mathrm{~N}(4 \mathrm{i})$ & 2.39 & $3.0463(19)$ & 133.7 \\
& $\mathrm{H}(2 \mathrm{~B})$ & 0.86 & $\mathrm{~N}(5 \mathrm{i})$ & 2.20 & $3.0435(18)$ & 166.0
\end{tabular}

Symmetry Operations:

$\begin{array}{rrrr}\text { i } & x & -1+y & z \\ \text { ii } & 3 / 2-x & -1 / 2+y & 3 / 2-z\end{array}$


Table so. Crystal Data, Experimental Details of the X-ray Diffraction Study and structure Solution.

(a) Crystal Parameters at $293 \mathrm{~K}$

from a least-squares fit of the setting angles of 25 reflections with $12.0^{\circ}<\theta<15.0^{\circ}$

Chem. Formula $\quad \mathrm{C}_{23} \mathrm{H}_{26} \mathrm{~N}_{6} \mathrm{NiO}$

$\mathrm{FW}=461.21$

Crystal Habit:

dark-brown plate

Crystal System: Orthorhombic

Space Group: $2{ }_{1}{ }^{2}{ }_{1}{ }_{1}$ (No. 19)

$a=13.9124(13) \AA$

$\mathrm{b}=25.076(2) \AA$

$C=6.4440(9) \AA$

$V=2248.1(4) \AA^{3}$ $\lambda=0.71073 \AA$

Crystal Dimensions:

$0.50 \times 0.15 \times 0.10 \mathrm{~mm}$

Absences: $h 00: h=2 n+1$

$0 \mathrm{k} 0: \mathrm{k}=2 \mathrm{n}+1$

$001: 1=2 \mathrm{n}+1$

$\mathrm{Z}=4$

$F(000)=968$

pcalc $=1.363 \mathrm{~g} \cdot \mathrm{cm}^{-3}$

$\mu=0.890 \mathrm{~mm}^{-1}$

(b) Measurement and treatment of Intensity Data

Instrument: Enraf-Nonius CAD4

Radiation: $\operatorname{MoK} \alpha(\lambda=0.71073 \AA)$ graphite monochromated

Detector window:

Height: $4.0 \mathrm{~mm}$

Width: $\quad 4.0 \mathrm{~mm}$

Scan mode: $\omega-2 \theta$

Scan width: $(0.80+0.35 \tan \theta)^{\circ}$
Scan speed: $0.91-8.24^{\circ} / \mathrm{min}$

$\operatorname{Max} 2 \theta: 54^{\circ}$

$$
\left(\begin{array}{llllll}
\mathrm{h} & -17,17 & \mathrm{k} & -32,32 & 1 & -8,8
\end{array}\right)
$$

Standards :

Intensity: 3 refl. every $2 \mathrm{~h}$

Orientation: 3 refl. every 400 data refl.

Take-off angle: $2.95^{\circ}$

No. of refl. collected: 5692 
Table so. (Continued)

(c) Treatment of Intensity Data

Reduction to $\mathrm{FO}^{2}$ and $\sigma\left(\mathrm{FO}^{2}\right)$, corrections for backgrounds, attenuator and LP in the usual manner [1]. No significative standard intensities variations $( \pm 0.2 \%)$. Semi-empirical absorption corrections from psi scans [2] $(\operatorname{Tmin}=0.9563, \operatorname{Tmax}=0.9950) .4898$ unique reflections $[$ Rint $=0.0241]$. No. of observed data $[I>2 \sigma(I)]: 3669$

(d) Structure Solution and Refinement

Direct methods using SHELXS-97 [3]. Full-matrix least-squares refinement using SHELXL-97 [4]. All non-H atoms anisotropic. H atoms found on a difference Fourier map, introduced in calculations with the riding model, except that bonded to $\mathrm{N}(2)$ atom allowed to vary, with Uiso equal to 1.1 times that of atom of attachment. Scattering factors were taken from "International Tables for Crystallography" [5]. Refinement on Fo ${ }^{2}$ for all reflections. Weighted R-factors $w R$ and goodnesses of fit $S$ are based on $\mathrm{FO}^{2}$, conventional R-factors $\mathrm{R}$ are based on Fo, with Fo set to zero for negative $\mathrm{Fo}^{2}$. Last refinement cycle: $\mathrm{wR}(\mathrm{all})=0.0541, \mathrm{R}(\mathrm{all})$ $=0.0334, \mathrm{wR}(\mathrm{obs})=0.0533, \mathrm{R}(\mathrm{obs})=0.0276$ with 3669 obs. reflections $>4 \sigma(\mathrm{FO}), 283$ variable parameters, $\mathrm{w}=\left[\sigma^{2}\left(\mathrm{FO}^{2}\right)+(0.0271 \mathrm{P})^{2}\right]^{-1}$ where $\mathrm{P}=\left(\mathrm{FO}^{2}+2 \mathrm{FC}^{2}\right) / 3, \mathrm{~S}=0.811,(\Delta / \sigma) \max =0.013,(\Delta / \sigma)$ mean $=0.001$, max. and min. residual peaks of 0.211 and $-0.156 \mathrm{e} / \AA^{3}$. Absolute configuration was determined: Flack coefficient [6] $=-0.012(11)$. 
Table so. (Continued)

[1] Fair, C.K. Molen. Structure Solution Procedures, Enraf-Nonius, Delft, Holland, 1990.

[2] North, A.C.T., Phillips, D.C. and Mathews, F.S. Acta Crystallogr., sect. A, 1968, A24, 351-359.

[3] Sheldrick, G.M. SHELXS-97. Program for Crystal Structure Solution. Univ. of Göttingen: Göttingen, Germany, 1990.

[4] Sheldrick, G.M. SHELXL-97. Program for the refinement of crystal structures from diffraction data. Univ. of Göttingen: Göttingen, Germany, 1997.

[5] "International Tables for Crystallography", Vol. C, Kluwer Academic Publishers, Dordrecht, The Netherlands, 1992, Tables 4.2.6.8 and 6.1.1.4.

[6] Flack, H.D. Acta Crystallogr., sect. A, 1983, A39, 876-881. 


\section{[ $\left.\left(\mathrm{C}_{23} \mathrm{H}_{26} \mathrm{~N}_{6} \mathrm{O}\right) \mathrm{Ni}\right]$}

Page 4

Table S1. Atomic coordinates and equivalent isotropic displacement parameters $\left(\AA^{2} \times 100\right)$.

\begin{tabular}{|c|c|c|c|c|}
\hline Atom & $\mathrm{x} / \mathrm{a}$ & $\mathrm{y} / \mathrm{b}$ & $z / c$ & Ueq ${ }^{*}$ \\
\hline $\mathrm{Ni}$ & $0.56514(2)$ & $0.30794(1)$ & $0.42617(4)$ & $3.736(6)$ \\
\hline$O(1)$ & $0.6031(1)$ & $0.34086(6)$ & $0.6660(2)$ & $4.27(3)$ \\
\hline $\mathrm{N}(1)$ & $0.6867(1)$ & $0.28263(6)$ & $0.3541(2)$ & $3.45(4)$ \\
\hline $\mathrm{N}(2)$ & $0.5249(1)$ & $0.27626(8)$ & $0.1846(3)$ & $4.72(5)$ \\
\hline $\mathrm{N}(3)$ & $0.8023(2)$ & $0.40849(8)$ & $1.1980(3)$ & $5.37(5)$ \\
\hline $\mathrm{N}(4)$ & $0.8336(2)$ & $0.20676(8)$ & $0.0302(4)$ & $6.76(7)$ \\
\hline$N(5)$ & $0.5563(2)$ & $0.2024(1)$ & $-0.2588(3)$ & $7.72(7)$ \\
\hline $\mathrm{N}(6)$ & $0.4370(1)$ & $0.33211(7)$ & $0.4953(3)$ & $4.60(4)$ \\
\hline$C(1)$ & $0.6904(1)$ & $0.34535(8)$ & $0.7394(3)$ & $3.76(4)$ \\
\hline$C(2)$ & $0.7032(2)$ & $0.37455(8)$ & $0.9216(4)$ & $4.07(4)$ \\
\hline$C(3)$ & $0.7928(2)$ & $0.38149(8)$ & $1.0135(3)$ & $4.33(5)$ \\
\hline$C(4)$ & $0.8735(2)$ & $0.35935(9)$ & $0.9107(4)$ & $5.14(6)$ \\
\hline$C(5)$ & $0.8625(2)$ & $0.33083(9)$ & $0.7361(4)$ & $4.79(6)$ \\
\hline$C(6)$ & $0.7720(1)$ & $0.32214(8)$ & $0.6412(3)$ & $3.64(5)$ \\
\hline$C(7)$ & $0.7655(2)$ & $0.29291(7)$ & $0.4585(4)$ & $4.01(5)$ \\
\hline$C(8)$ & $0.7183(2)$ & $0.4290(1)$ & $1.3066(4)$ & $5.65(7)$ \\
\hline C (9) & $0.6817(2)$ & $0.4804(1)$ & $1.2261(4)$ & $7.21(8)$ \\
\hline$C(10)$ & $0.8948(2)$ & $0.4158(1)$ & $1.2987(4)$ & $6.13(7)$ \\
\hline$C(11)$ & $0.9494(2)$ & $0.4636(1)$ & $1.2221(5)$ & $7.53(8)$ \\
\hline$C(12)$ & $0.6854(2)$ & $0.25219(8)$ & $0.1713(3)$ & $3.84(5)$ \\
\hline$C(13)$ & $0.5946(2)$ & $0.25064(8)$ & $0.0859(4)$ & $4.25(5)$ \\
\hline$C(14)$ & $0.7664(2)$ & $0.22748(9)$ & $0.0902(4)$ & $4.71(5)$ \\
\hline$C(15)$ & $0.5755(2)$ & $0.2239(1)$ & $-0.1088(3)$ & $5.44(6)$ \\
\hline$C(16)$ & $0.4167(2)$ & $0.38986(9)$ & $0.4828(4)$ & $4.83(6)$ \\
\hline$C(17)$ & $0.3211(2)$ & $0.40270(9)$ & $0.5803(4)$ & $4.81(5)$ \\
\hline$C(18)$ & $0.3149(2)$ & $0.4360(1)$ & $0.7475(4)$ & $6.18(7)$ \\
\hline$C(19)$ & $0.2265(3)$ & $0.4474(1)$ & $0.8398(5)$ & $8.6(1)$ \\
\hline$C(20)$ & $0.1465(2)$ & $0.4259(1)$ & $0.7652(6)$ & $8.6(1)$ \\
\hline$C(21)$ & $0.1500(2)$ & $0.3916(1)$ & $0.6037(6)$ & $8.4(1)$ \\
\hline$C(22)$ & $0.2365(2)$ & $0.3805(1)$ & $0.5109(5)$ & $6.63(8)$ \\
\hline$C(23)$ & $0.4226(2)$ & $0.4075(1)$ & $0.2596(4)$ & $6.90(8)$ \\
\hline
\end{tabular}

Ueq $=1 / 3$ of the trace of the orthogonalized Uij tensor. 
Table s2. Hydrogen atomic positional and thermal parameters $\left(\AA^{2} \times 100\right)$.

\begin{tabular}{lllll}
\hline Atom & x/a & $y / b$ & $z / C$ & $U$ \\
\hline H (2A) & $0.4716(14)$ & $0.2680(9)$ & $0.1480(41)$ & 5.2 \\
H (6A) & 0.3954 & 0.3152 & 0.4109 & 5.1 \\
H (6B) & 0.4241 & 0.3214 & 0.6256 & 5.1 \\
H (2) & 0.6498 & 0.3900 & 0.9841 & 4.5 \\
H (4) & 0.9348 & 0.3646 & 0.9648 & 5.7 \\
H (5) & 0.9168 & 0.3161 & 0.6745 & 5.3 \\
H (7) & 0.8225 & 0.2793 & 0.4050 & 4.4 \\
H (8A) & 0.7344 & 0.4334 & 1.4521 & 6.2 \\
H (8B) & 0.6673 & 0.4027 & 1.2978 & 6.2 \\
H (9A) & 0.6281 & 0.4919 & 1.3087 & 7.9 \\
H (9B) & 0.6615 & 0.4761 & 1.0847 & 7.9 \\
H (9C) & 0.7317 & 0.5067 & 1.2327 & 7.9 \\
H (10A) & 0.9335 & 0.3842 & 1.2764 & 6.7 \\
H (10B) & 0.8846 & 0.4194 & 1.4469 & 6.7 \\
H (11A) & 1.0099 & 0.4658 & 1.2933 & 8.3 \\
H (11B) & 0.9127 & 0.4953 & 1.2482 & 8.3 \\
H (11C) & 0.9606 & 0.4601 & 1.0757 & 8.3 \\
H (16) & 0.4667 & 0.4087 & 0.5609 & 5.3 \\
H (18) & 0.3705 & 0.4513 & 0.8009 & 6.8 \\
H (19) & 0.2236 & 0.4701 & 0.9536 & 9.4 \\
H (20) & 0.0876 & 0.4345 & 0.8244 & 9.4 \\
H (21) & 0.0940 & 0.3755 & 0.5556 & 9.3 \\
H (22) & 0.2380 & 0.3574 & 0.3982 & 7.3 \\
H (23A) & 0.4848 & 0.3987 & 0.2047 & 7.6 \\
H (23B) & 0.4129 & 0.4453 & 0.2516 & 7.6 \\
H (23C) & 0.3739 & 0.3896 & 0.1802 & 7.6 \\
& & & & \\
\hline
\end{tabular}


Table s3. Final anisotropic displacement parameters $\left(\AA^{2} \times 100\right)$.

\begin{tabular}{|c|c|c|c|c|c|c|}
\hline Atom & U11 & U22 & U33 & U12 & U13 & U23 \\
\hline $\mathrm{Ni}$ & $3.11(1)$ & $4.46(1)$ & $3.65(1)$ & $-0.23(1)$ & $0.12(1)$ & $0.16(1)$ \\
\hline$O(1)$ & $3.33(8)$ & $5.36(9)$ & $4.13(8)$ & $-0.73(7)$ & $0.50(6)$ & $-0.08(7)$ \\
\hline $\mathrm{N}(1)$ & $3.16(9)$ & $3.55(8)$ & $3.66(9)$ & $0.39(7)$ & $0.36(7)$ & $0.22(7)$ \\
\hline $\mathrm{N}(2)$ & $3.6(1)$ & $6.0(1)$ & $4.6(1)$ & $-0.52(9)$ & $-0.13(9)$ & $-0.15(9)$ \\
\hline$N(3)$ & $5.4(1)$ & $6.2(1)$ & $4.5(1)$ & $-0.6(1)$ & $-1.0(1)$ & $-0.8(1)$ \\
\hline $\mathrm{N}(4)$ & $5.5(1)$ & $6.3(1)$ & $8.4(2)$ & $-1.0(1)$ & $3.2(1)$ & $0.0(1)$ \\
\hline $\mathrm{N}(5)$ & $9.1(2)$ & $8.7(2)$ & $5.4(1)$ & $-2.0(1)$ & $0.1(1)$ & $-2.5(2)$ \\
\hline $\mathrm{N}(6)$ & $3.36(9)$ & $5.6(1)$ & $4.88(9)$ & $-0.29(8)$ & $-0.16(9)$ & $0.1(1)$ \\
\hline$C(1)$ & $3.1(1)$ & $4.2(1)$ & $4.0(1)$ & $0.63(9)$ & $-0.41(9)$ & $-0.47(9)$ \\
\hline$C(2)$ & $4.0(1)$ & $4.7(1)$ & $3.48(9)$ & $-0.1(1)$ & $0.6(1)$ & $0.01(9)$ \\
\hline$C(3)$ & $5.4(1)$ & $3.6(1)$ & $3.9(1)$ & $0.43(9)$ & $-0.7(1)$ & $-1.0(1)$ \\
\hline$C(4)$ & $3.5(1)$ & $6.1(1)$ & $5.8(1)$ & $1.0(1)$ & $-1.1(1)$ & $-0.1(1)$ \\
\hline$C(5)$ & $3.8(1)$ & $5.3(1)$ & $5.3(1)$ & $0.0(1)$ & $-0.4(1)$ & $0.9(1)$ \\
\hline$C(6)$ & $3.1(1)$ & $4.2(1)$ & $3.7(1)$ & $0.06(8)$ & $-0.21(8)$ & $0.48(8)$ \\
\hline$C(7)$ & $3.1(1)$ & $4.1(1)$ & $4.8(1)$ & $0.54(9)$ & $-0.15(9)$ & $0.41(8)$ \\
\hline$C(8)$ & $7.2(2)$ & $5.6(1)$ & $4.2(1)$ & $-0.4(1)$ & $-0.6(1)$ & $-1.0(1)$ \\
\hline$C(9)$ & $8.9(2)$ & $7.3(2)$ & $5.4(2)$ & $0.0(1)$ & $0.5(2)$ & $0.1(2)$ \\
\hline$C(10)$ & $6.8(2)$ & $7.2(2)$ & $4.4(1)$ & $-0.4(1)$ & $-0.7(1)$ & $-1.1(1)$ \\
\hline$C(11)$ & $7.3(2)$ & $8.6(2)$ & $6.8(2)$ & $-0.5(2)$ & $-0.9(2)$ & $-2.2(2)$ \\
\hline$C(12)$ & $4.0(1)$ & $3.8(1)$ & $3.7(1)$ & $-0.62(9)$ & $0.81(9)$ & $0.23(9)$ \\
\hline$C(13)$ & $4.7(1)$ & $4.3(1)$ & $3.8(1)$ & $0.0(1)$ & $0.1(1)$ & $-0.81(9)$ \\
\hline$C(14)$ & $4.7(1)$ & $4.8(1)$ & $4.6(1)$ & $-0.5(1)$ & $1.2(1)$ & $-0.4(1)$ \\
\hline$C(15)$ & $5.4(2)$ & $6.5(1)$ & $4.4(1)$ & $-0.7(1)$ & $0.6(1)$ & $-1.7(1)$ \\
\hline$C(16)$ & $4.0(1)$ & $4.9(1)$ & $5.6(1)$ & $-0.9(1)$ & $-0.1(1)$ & $0.3(1)$ \\
\hline$C(17)$ & $4.8(1)$ & $5.5(1)$ & $4.2(1)$ & $-0.2(1)$ & $0.0(1)$ & $0.7(1)$ \\
\hline$C(18)$ & $6.8(2)$ & $6.4(2)$ & $5.3(2)$ & $-0.8(1)$ & $-0.6(1)$ & $1.0(1)$ \\
\hline$C(19)$ & $11.1(3)$ & $8.4(2)$ & $6.2(2)$ & $-1.9(2)$ & $2.2(2)$ & $3.4(2)$ \\
\hline$C(20)$ & $6.3(2)$ & $10.1(3)$ & $9.2(3)$ & $-1.1(2)$ & $2.3(2)$ & $1.9(2)$ \\
\hline$C(21)$ & $4.3(2)$ & $10.7(2)$ & $10.4(3)$ & $-2.1(2)$ & $0.9(2)$ & $1.0(2)$ \\
\hline$C(22)$ & $4.8(1)$ & $7.8(2)$ & $7.3(2)$ & $-2.6(2)$ & $0.1(1)$ & $1.4(1)$ \\
\hline$C(23)$ & $7.2(2)$ & $7.0(2)$ & $6.5(2)$ & $1.4(1)$ & $0.9(2)$ & $1.4(2)$ \\
\hline
\end{tabular}


Table s4. Bond lengths ( $\AA$ ) and angles (deg) with e.s.d.'s in parentheses.

\section{Bond Lengths}

$$
\begin{aligned}
& \mathrm{Ni}-\mathrm{O}(1) \\
& \mathrm{Ni}-\mathrm{N}(1) \\
& \mathrm{O}(1)-\mathrm{C}(1) \\
& \mathrm{C}(1)-\mathrm{C}(2) \\
& \mathrm{C}(2)-\mathrm{C}(3) \\
& \mathrm{C}(3)-\mathrm{C}(4) \\
& \mathrm{C}(3)-\mathrm{N}(3) \\
& \mathrm{C}(4)-\mathrm{C}(5) \\
& \mathrm{C}(5)-\mathrm{C}(6)
\end{aligned}
$$

$\mathrm{N}(2)-\mathrm{C}(13)$

$C(12)-C(13)$

$C(13)-C(15)$

$\mathrm{C}(15)-\mathrm{N}(5)$

$C(16)-C(17)$

$C(17)-C(18)$

$C(18)-C(19)$

$C(19)-C(20)$

\section{$1.8295(15)$}

1.8647 (17)

$1.308(2)$

$1.395(3)$

$1.390(3)$

$1.417(3)$

$1.375(3)$

$1.342(3)$

$1.417(3)$

$\mathrm{N}(1)-\mathrm{C}(12)$

$1.326(3)$
$1.379(3)$
$1.448(3)$
$1.138(3)$
$1.506(3)$
$1.365(3)$
$1.396(4)$
$1.327(4)$

Bond Angles

$\mathrm{O}(1)-\mathrm{Ni}-\mathrm{N}(1)$

$\mathrm{O}(1)-\mathrm{Ni}-\mathrm{N}(2)$

$\mathrm{O}(1)-\mathrm{Ni}-\mathrm{N}(6)$

$\mathrm{Ni}-\mathrm{O}(1)-\mathrm{C}(1)$

$O(1)-C(1)-C(6)$

$O(1)-C(1)-C(2)$

$C(6)-C(1)-C(2)$

$C(1)-C(2)-C(3)$

$C(2)-C(3)-C(4)$

$\mathrm{C}(2)-\mathrm{C}(3)-\mathrm{N}(3)$

$\mathrm{C}(4)-\mathrm{C}(3)-\mathrm{N}(3)$

$\mathrm{C}(3)-\mathrm{N}(3)-\mathrm{C}(8)$

$\mathrm{C}(3)-\mathrm{N}(3)-\mathrm{C}(10)$

$\mathrm{C}(8)-\mathrm{N}(3)-\mathrm{C}(10)$

$\mathrm{Ni}-\mathrm{N}(2)-\mathrm{H}(2 \mathrm{~A})$

$\mathrm{Ni}-\mathrm{N}(2)-\mathrm{C}(13)$

$\mathrm{C}(13)-\mathrm{N}(2)-\mathrm{H}(2 \mathrm{~A})$
$129.9(16)$

$95.88(7)$

$178.56(8)$

$85.98(7)$

$127.74(14)$

$122.95(19)$

117.94 (19)

119.11 (19)

$122.6(2)$

$117.5(2)$

$121.1(2)$

$121.4(2)$

$120.8(2)$

$122.3(2)$

$116.9(2)$

$114.1(16)$
$\mathrm{Ni}-\mathrm{N}(2)$

$\mathrm{Ni}-\mathrm{N}(6)$

$1.835(2)$

$1.9349(18)$

$C(6)-C(1)$

$C(6)-C(7)$

$1.424(3)$

$1.390(3)$

$1.312(3)$

$1.456(3)$

$1.452(3)$

$1.481(3)$

$1.502(4)$

$C(10)-C(11)$

$1.404(2)$

$\mathrm{N}(2)-\mathrm{H}(2 \mathrm{~A})$
$\mathrm{C}(12)-\mathrm{C}(14)$
$\mathrm{C}(14)-\mathrm{N}(4)$
$\mathrm{N}(6)-\mathrm{C}(16)$
$\mathrm{C}(16)-\mathrm{C}(23)$
$\mathrm{C}(20)-\mathrm{C}(21)$
$\mathrm{C}(21)-\mathrm{C}(22)$
$\mathrm{C}(22)-\mathrm{C}(17)$

$0.81(2)$

$1.388(3)$

$1.137(3)$

$1.478(3)$

$1.507(3)$

$1.351(4)$

$1.372(3)$

$1.377(3)$
$\mathrm{N}(1)-\mathrm{Ni}-\mathrm{N}(2)$

$\mathrm{N}(1)-\mathrm{Ni}-\mathrm{N}(6)$

$\mathrm{N}(2)-\mathrm{Ni}-\mathrm{N}(6)$

$\mathrm{N}(3)-\mathrm{C}(8)-\mathrm{C}(9)$

$\mathrm{N}(3)-\mathrm{C}(10)-\mathrm{C}(11)$

$C(3)-C(4)-C(5)$

$C(4)-C(5)-C(6)$

$C(5)-C(6)-C(1)$

$C(5)-C(6)-C(7)$

$C(1)-C(6)-C(7)$

$\mathrm{C}(6)-\mathrm{C}(7)-\mathrm{N}(1)$

$\mathrm{Ni}-\mathrm{N}(1)-\mathrm{C}(7)$

$\mathrm{Ni}-\mathrm{N}(1)-\mathrm{C}(12)$

$\mathrm{C}(7)-\mathrm{N}(1)-\mathrm{C}(12)$

$113.18(16)$

$\mathrm{N}(6)-\mathrm{C}(16)-\mathrm{C}(17)$

$\mathrm{N}(6)-\mathrm{C}(16)-\mathrm{C}(23)$
$85.31(8)$

$177.93(8)$

$92.84(8)$

$114.6(2)$

$113.7(2)$

$120.7(2)$

$123.0(2)$

116.99 (19)

120.28 (19)

122.72 (19)

$126.3(2)$

124.29 (14)

$112.52(14)$

$123.18(18)$

$110.83(19)$

$109.20(19)$ 
Table s4. (Continued)

\begin{tabular}{|c|c|c|c|c|c|}
\hline $\begin{array}{l}\mathrm{N}(1)-\mathrm{C}(12)-\mathrm{C}(13) \\
\mathrm{N}(1)-\mathrm{C}(12)-\mathrm{C}(14) \\
\mathrm{C}(13)-\mathrm{C}(12)-\mathrm{C}(14) \\
\mathrm{N}(2)-\mathrm{C}(13)-\mathrm{C}(12) \\
\mathrm{N}(2)-\mathrm{C}(13)-\mathrm{C}(15) \\
\mathrm{C}(12)-\mathrm{C}(13)-\mathrm{C}(15) \\
\mathrm{C}(12)-\mathrm{C}(14)-\mathrm{N}(4) \\
\mathrm{C}(13)-\mathrm{C}(15)-\mathrm{N}(5) \\
\mathrm{Ni}-\mathrm{N}(6)-\mathrm{C}(16)\end{array}$ & \multicolumn{2}{|c|}{$\begin{array}{l}111.19(18) \\
123.3(2) \\
125.6(2) \\
117.7(2) \\
120.4(2) \\
121.8(2) \\
177.8(3) \\
176.9(3) \\
118.06(14)\end{array}$} & \multicolumn{2}{|c|}{$\begin{array}{l}C(17)-C(16)-C(23) \\
C(16)-C(17)-C(22) \\
C(16)-C(17)-C(18) \\
C(22)-C(17)-C(18) \\
C(17)-C(18)-C(19) \\
C(18)-C(19)-C(20) \\
C(19)-C(20)-C(21) \\
C(20)-C(21)-C(22) \\
C(21)-C(22)-C(17)\end{array}$} & \multirow[t]{2}{*}{$\begin{array}{l}112.5(2) \\
122.2(2) \\
121.0(2) \\
116.7(2) \\
121.2(3) \\
120.1(3) \\
120.5(3) \\
119.7(3) \\
121.8(3)\end{array}$} \\
\hline \multicolumn{5}{|l|}{ Hydrogen Bonds } & \\
\hline $\mathrm{H}$ & $\mathrm{D}-\mathrm{H}$ & $\mathrm{A}$ & $\mathrm{H} \ldots \mathrm{A}$ & $D \ldots A$ & $\mathrm{D}-\mathrm{H} \ldots \mathrm{A}$ \\
\hline $\mathrm{H}(2 \mathrm{~A})$ & $0.81(2)$ & $N\left(4^{\prime}\right)$ & $2.32(2)$ & $3.029(3)$ & $147(2)$ \\
\hline Symmetry Operatio & ons : & $-1 / 2$ & $+x$ & $2-y$ & $-z$ \\
\hline
\end{tabular}


Table S5. Least-squares planes equations and deviations

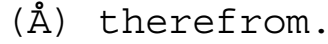

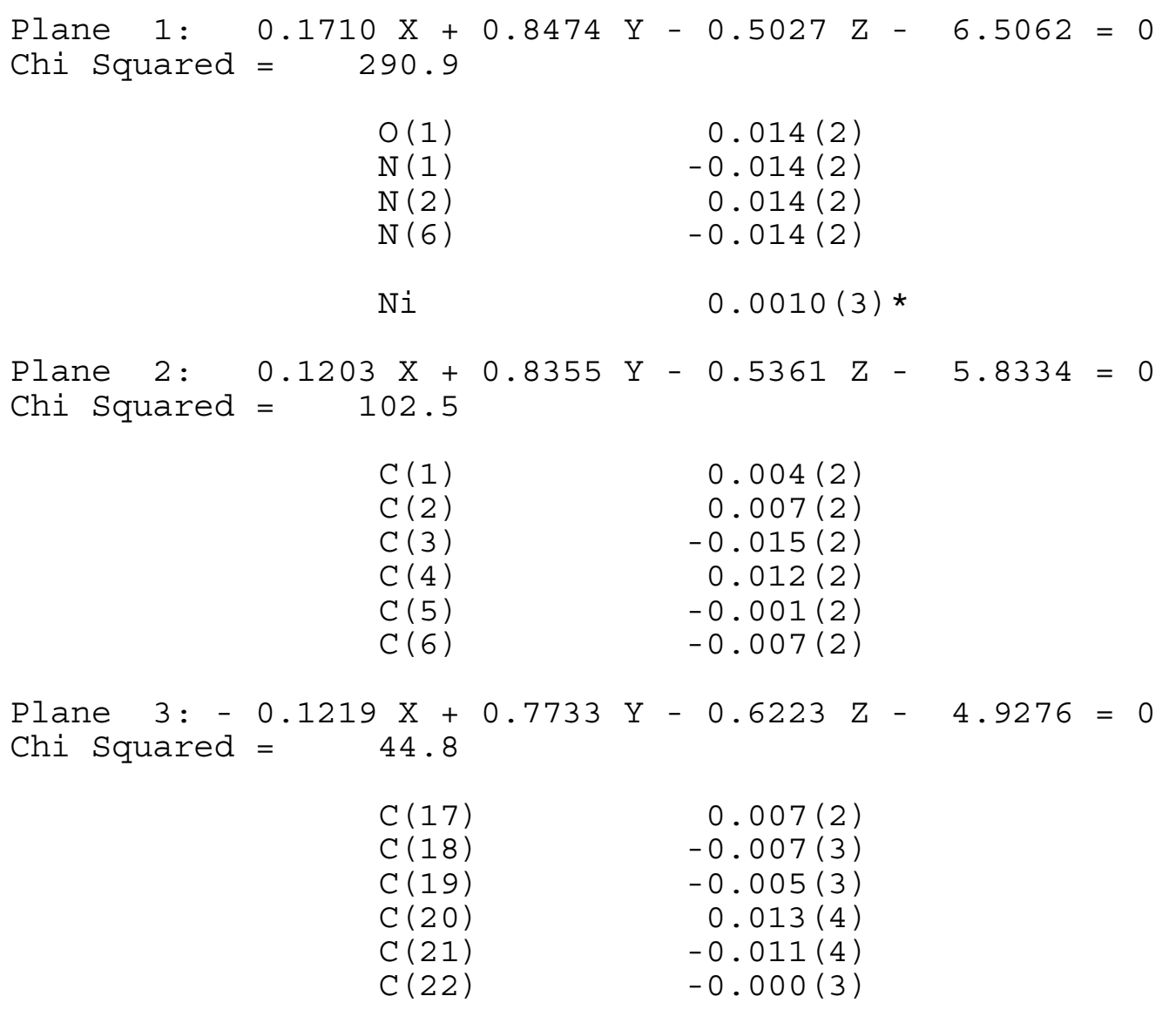

* denote atoms not included in planes calculations. 
Table so. Crystal Data, Experimental Details of the X-ray Diffraction Study and structure Solution.

(a) Crystal Parameters at $180 \mathrm{~K}$

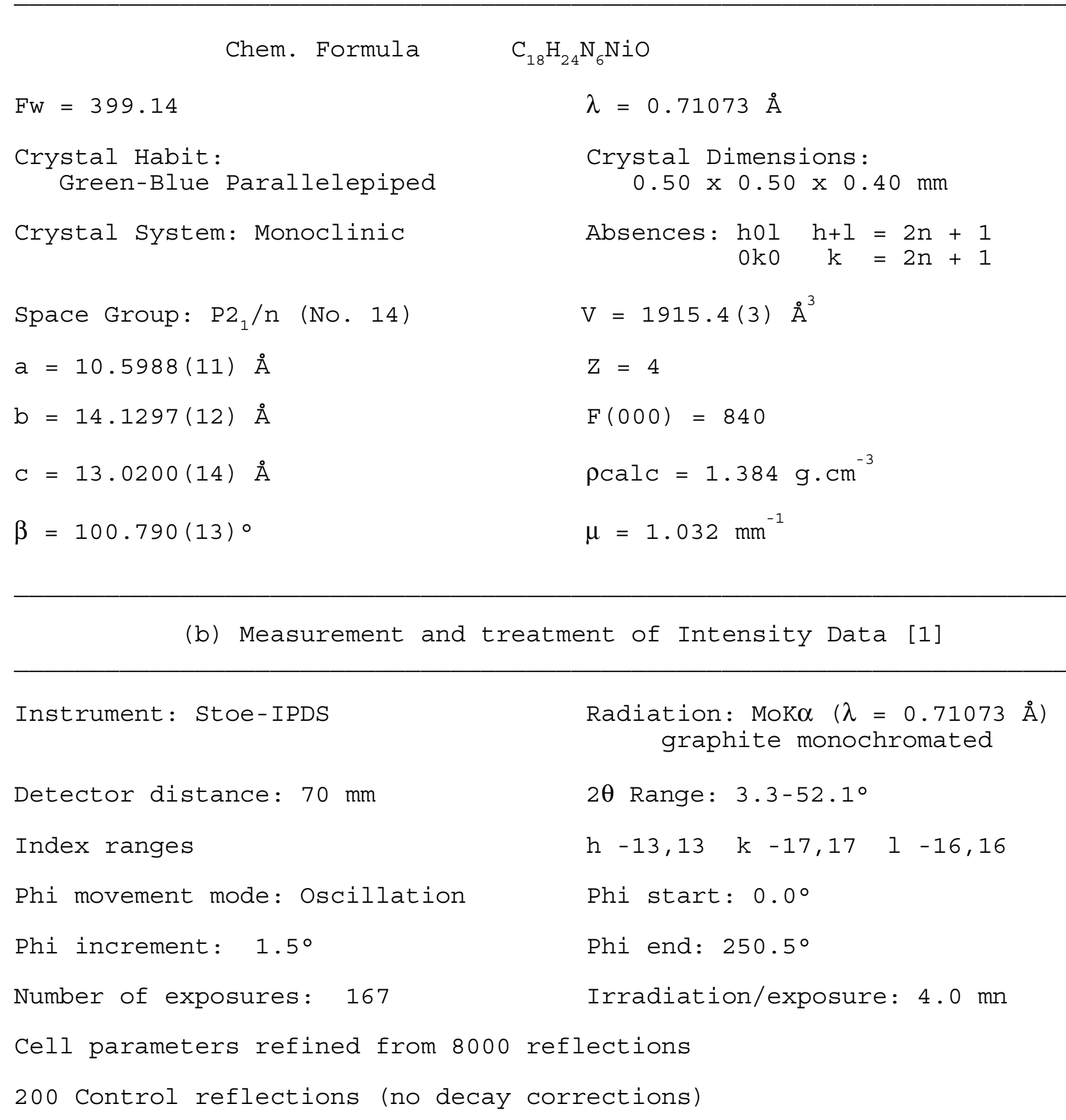


Table so. (Continued)

Integration:

Profile function: Dynamic Reflections collected: 18357

Absorption corrections [2]
Effective mosaic spread: 0.010

Independent reflections: 3710 $[R($ int $)=0.0334]$

$\operatorname{Tmin}=0.5805, \operatorname{Tmax}=0.7691$

(c) Structure Solution and Refinement

Direct methods using SHELXS-97 [3]. Full-matrix least-squares refinement using SHELXL-97 [4]. All non-H atoms anisotropic. H atoms found on a difference Fourier map, introduced in calculations with the riding model, with isotropic U equal to 1.1 times that of atom of attachment. Scattering factors were taken from "International Tables for Crystallography" [5]. Refinement on $\mathrm{FO}^{2}$ for all reflections. Weighted R-factors $w R$ and goodness of fit $S$ are based on $\mathrm{FO}^{2}$, conventional Rfactors $R$ are based on Fo, with Fo set to zero for negative Fo ${ }^{2}$. Last refinement cycle: $\mathrm{wR}(\mathrm{all})=0.0539, \mathrm{R}(\mathrm{all})=0.0276, \mathrm{wR}(\mathrm{obs})=0.0525$, $\mathrm{R}(\mathrm{Obs})=0.0248$ with 3375 obs. reflections $>4 \sigma(\mathrm{Fo}), 235$ variable parameters, $\mathrm{w}=\left[\sigma^{2}\left(\mathrm{FO}^{2}\right)+(0.0374 \mathrm{P})^{2}+0.5115 \mathrm{P}\right]^{-1}$ where $\mathrm{P}=\left(\mathrm{FO}^{2}+\right.$ $\left.2 \mathrm{FC}^{2}\right) / 3, \quad \mathrm{~S}=1.050,(\Delta / \sigma) \max =0.001,(\Delta / \sigma)$ mean $=0.000$, max. and $\min$. residual peaks of 0.259 and $-0.324 \mathrm{e} / \AA^{3}$. 
Table so. (Continued)

[1] Stoe, IPDS Manual. Version 2.87. Stoe \& Cie, Darmstadt, Germany, 1997.

[2] Stoe, X-SHAPE, Crystal Optimisation for Numerical Absorption Correction, Revision 1.01. Stoe \& Cie, Darmstadt, Germany, 1996.

[3] Sheldrick, G.M. SHELXS-97. Program for Crystal Structure Solution. Univ. of Göttingen: Göttingen, Germany, 1990.

[4] Sheldrick, G.M. SHELXL-97. Program for the refinement of crystal structures from diffraction data. Univ. of Göttingen: Göttingen, Germany, 1997.

[5] "International Tables for Crystallography", Vol. C, Kluwer Academic Publishers, Dordrecht, The Netherlands, 1992, Tables 4.2 .6 .8 and 6.1 .1 .4 . 
Table S1. Atomic coordinates and equivalent isotropic displacement parameters $\left(\AA^{2} \times 100\right)$.

\begin{tabular}{|c|c|c|c|c|}
\hline Atom & $\mathrm{x} / \mathrm{a}$ & $y / b$ & $\mathrm{z} / \mathrm{C}$ & Ueq ${ }^{*}$ \\
\hline $\mathrm{Ni}$ & $0.90103(1)$ & $0.28597(1)$ & $0.41960(1)$ & $2.08(1)$ \\
\hline 0 & $0.95534(9)$ & $0.25394(7)$ & $0.55678(7)$ & $2.58(2)$ \\
\hline $\mathrm{N}(1)$ & $0.8567(1)$ & $0.41035(8)$ & $0.44560(8)$ & $2.13(2)$ \\
\hline$N(2)$ & $0.8379(1)$ & $0.31357(9)$ & $0.28097(9)$ & $2.72(3)$ \\
\hline $\mathrm{N}(3)$ & $0.9565(1)$ & $0.16130(8)$ & $0.38795(9)$ & $2.69(3)$ \\
\hline$N(4)$ & $1.0236(1)$ & $0.27091(9)$ & $0.92593(9)$ & $3.16(3)$ \\
\hline$N(5)$ & $0.7276(1)$ & $0.63083(9)$ & $0.3519(1)$ & $3.31(3)$ \\
\hline$N(6)$ & $0.6866(2)$ & $0.4611(1)$ & $0.0827(1)$ & $5.06(4)$ \\
\hline$C(1)$ & $0.9555(1)$ & $0.3068(1)$ & $0.6392(1)$ & $2.22(3)$ \\
\hline$C(2)$ & $0.9891(1)$ & $0.2639(1)$ & $0.7377(1)$ & $2.50(3)$ \\
\hline$C(3)$ & $0.9960(1)$ & $0.3146(1)$ & $0.8309(1)$ & $2.58(3)$ \\
\hline$C(4)$ & $0.9711(1)$ & $0.4141(1)$ & $0.8234(1)$ & $2.86(3)$ \\
\hline$C(5)$ & $0.9342(1)$ & $0.4556(1)$ & $0.7282(1)$ & $2.63(3)$ \\
\hline$C(6)$ & $0.9214(1)$ & $0.40450(9)$ & $0.6331(1)$ & $2.25(3)$ \\
\hline$C(7)$ & $0.8748(1)$ & $0.45097(9)$ & $0.5379(1)$ & $2.23(3)$ \\
\hline$C(8)$ & $0.8026(1)$ & $0.45837(9)$ & $0.3533(1)$ & $2.29(3)$ \\
\hline C (9) & $0.7923(1)$ & $0.4013(1)$ & $0.2663(1)$ & $2.49(3)$ \\
\hline$C(10)$ & $1.0542(1)$ & $0.1560(1)$ & $0.3197(1)$ & $3.47(3)$ \\
\hline$C(11)$ & $1.1685(2)$ & $0.2158(1)$ & $0.3660(2)$ & $5.64(5)$ \\
\hline$C(12)$ & $1.0904(2)$ & $0.0539(1)$ & $0.3033(2)$ & $4.62(4)$ \\
\hline$C(13)$ & $1.0218(2)$ & $0.3198(1)$ & $1.0249(1)$ & $3.80(4)$ \\
\hline$C(14)$ & $1.1528(2)$ & $0.3583(2)$ & $1.0740(2)$ & $5.94(6)$ \\
\hline$C(15)$ & $1.0473(2)$ & $0.1694(1)$ & $0.9343(1)$ & $3.71(4)$ \\
\hline$C(16)$ & $0.9245(2)$ & $0.1119(1)$ & $0.9191(1)$ & $4.64(4)$ \\
\hline$C(17)$ & $0.7624(1)$ & $0.5538(1)$ & $0.3517(1)$ & $2.44(3)$ \\
\hline$C(18)$ & $0.7335(2)$ & $0.4349(1)$ & $0.1641(1)$ & $3.18(3)$ \\
\hline
\end{tabular}


Table s2. Hydrogen atomic positional and thermal parameters $\left(\AA^{2} \times 100\right)$.

\begin{tabular}{|c|c|c|c|c|}
\hline Atom & $\mathrm{x} / \mathrm{a}$ & $\mathrm{y} / \mathrm{b}$ & $z / C$ & $\mathrm{U}$ \\
\hline $\mathrm{H}(2 \mathrm{~A})$ & 0.8379 & 0.2729 & 0.2298 & 3.0 \\
\hline $\mathrm{H}(3 \mathrm{~A})$ & 0.9892 & 0.1315 & 0.4500 & 3.0 \\
\hline $\mathrm{H}(3 \mathrm{~B})$ & 0.8854 & 0.1276 & 0.3564 & 3.0 \\
\hline $\mathrm{H}(2)$ & 1.0079 & 0.1981 & 0.7412 & 2.8 \\
\hline $\mathrm{H}(4)$ & 0.9804 & 0.4514 & 0.8851 & 3.1 \\
\hline $\mathrm{H}(5)$ & 0.9162 & 0.5215 & 0.7253 & 2.9 \\
\hline $\mathrm{H}(7)$ & 0.8549 & 0.5164 & 0.5407 & 2.5 \\
\hline H (10) & 1.0155 & 0.1833 & 0.2500 & 3.8 \\
\hline $\mathrm{H}(11 \mathrm{~A})$ & 1.1403 & 0.2809 & 0.3747 & 6.2 \\
\hline $\mathrm{H}(11 \mathrm{~B})$ & 1.2309 & 0.2154 & 0.3192 & 6.2 \\
\hline $\mathrm{H}(11 \mathrm{C})$ & 1.2085 & 0.1901 & 0.4342 & 6.2 \\
\hline $\mathrm{H}(12 \mathrm{~A})$ & 1.0132 & 0.0181 & 0.2729 & 5.1 \\
\hline $\mathrm{H}(12 \mathrm{~B})$ & 1.1295 & 0.0259 & 0.3706 & 5.1 \\
\hline $\mathrm{H}(12 \mathrm{C})$ & 1.1519 & 0.0517 & 0.2557 & 5.1 \\
\hline $\mathrm{H}(13 \mathrm{~A})$ & 0.9925 & 0.2752 & 1.0742 & 4.2 \\
\hline $\mathrm{H}(13 \mathrm{~B})$ & 0.9596 & 0.3726 & 1.0123 & 4.2 \\
\hline $\mathrm{H}(14 \mathrm{~A})$ & 1.1471 & 0.3905 & 1.1396 & 6.5 \\
\hline $\mathrm{H}(14 \mathrm{~B})$ & 1.1817 & 0.4032 & 1.0259 & 6.5 \\
\hline $\mathrm{H}(14 \mathrm{C})$ & 1.2144 & 0.3060 & 1.0883 & 6.5 \\
\hline $\mathrm{H}(15 \mathrm{~A})$ & 1.0992 & 0.1552 & 1.0041 & 4.1 \\
\hline $\mathrm{H}(15 \mathrm{~B})$ & 1.0980 & 0.1502 & 0.8811 & 4.1 \\
\hline $\mathrm{H}(16 \mathrm{~A})$ & 0.9457 & 0.0443 & 0.9256 & 5.1 \\
\hline $\mathrm{H}(16 \mathrm{~B})$ & 0.8737 & 0.1244 & 0.8495 & 5.1 \\
\hline $\mathrm{H}(16 \mathrm{C})$ & 0.8747 & 0.1296 & 0.9725 & 5.1 \\
\hline
\end{tabular}


Table s3. Final anisotropic displacement parameters $\left(\AA^{2} \mathrm{x} 100\right)$.

\begin{tabular}{|c|c|c|c|c|c|c|}
\hline Atom & U11 & $\mathrm{U} 22$ & U33 & U12 & U13 & U23 \\
\hline $\mathrm{Ni}$ & $2.24(1)$ & $1.99(1)$ & $2.04(1)$ & $-0.16(1)$ & $0.45(1)$ & $0.02(1)$ \\
\hline 0 & $3.23(5)$ & $2.28(5)$ & $2.24(5)$ & $-0.13(4)$ & $0.56(4)$ & $0.41(4)$ \\
\hline $\mathrm{N}(1)$ & $1.88(5)$ & $2.22(6)$ & $2.31(5)$ & $0.07(4)$ & $0.44(4)$ & $-0.18(4)$ \\
\hline $\mathrm{N}(2)$ & $3.17(6)$ & $2.63(6)$ & $2.32(6)$ & $-0.35(5)$ & $0.46(5)$ & $0.12(5)$ \\
\hline$N(3)$ & $3.15(6)$ & $2.44(6)$ & $2.54(6)$ & $-0.31(5)$ & $0.66(5)$ & $0.09(5)$ \\
\hline $\mathrm{N}(4)$ & $3.68(7)$ & $3.67(7)$ & $2.12(6)$ & $-0.01(5)$ & $0.49(5)$ & $0.61(5)$ \\
\hline$N(5)$ & $3.88(7)$ & $2.74(7)$ & $3.23(7)$ & $0.45(5)$ & $0.51(5)$ & $0.50(5)$ \\
\hline $\mathrm{N}(6)$ & $7.1(1)$ & $4.90(9)$ & $2.87(7)$ & $0.23(6)$ & $0.13(7)$ & $1.61(8)$ \\
\hline$C(1)$ & $1.71(6)$ & $2.66(7)$ & $2.32(6)$ & $-0.17(5)$ & $0.50(5)$ & $-0.11(5)$ \\
\hline$C(2)$ & $2.36(6)$ & $2.70(7)$ & $2.53(7)$ & $0.00(6)$ & $0.65(5)$ & $0.29(5)$ \\
\hline$C(3)$ & $1.97(6)$ & $3.45(8)$ & $2.35(7)$ & $0.05(6)$ & $0.44(5)$ & $0.08(5)$ \\
\hline$C(4)$ & $2.99(7)$ & $3.22(8)$ & $2.37(7)$ & $-0.67(6)$ & $0.53(5)$ & $-0.15(6)$ \\
\hline$C(5)$ & $2.68(7)$ & $2.56(7)$ & $2.66(7)$ & $-0.45(6)$ & $0.58(5)$ & $-0.04(5)$ \\
\hline$C(6)$ & $1.89(6)$ & $2.46(7)$ & $2.46(6)$ & $-0.19(5)$ & $0.51(5)$ & $-0.17(5)$ \\
\hline$C(7)$ & $1.98(6)$ & $2.07(6)$ & $2.70(7)$ & $-0.24(5)$ & $0.59(5)$ & $-0.14(5)$ \\
\hline$C(8)$ & $2.15(6)$ & $2.33(7)$ & $2.41(6)$ & $0.22(5)$ & $0.48(5)$ & $-0.14(5)$ \\
\hline C (9) & $2.34(6)$ & $2.79(7)$ & $2.38(7)$ & $0.11(5)$ & $0.50(5)$ & $-0.15(5)$ \\
\hline$C(10)$ & $2.93(7)$ & $4.18(9)$ & $3.41(8)$ & $-0.12(7)$ & $0.86(6)$ & $0.66(6)$ \\
\hline$C(11)$ & $3.31(9)$ & $5.0(1)$ & $8.8(2)$ & $-0.4(1)$ & $1.6(1)$ & $-0.08(8)$ \\
\hline$C(12)$ & $4.03(9)$ & $5.0(1)$ & $4.9(1)$ & $-1.60(8)$ & $0.84(7)$ & $1.26(8)$ \\
\hline$C(13)$ & $4.42(9)$ & $4.7(1)$ & $2.37(7)$ & $0.02(7)$ & $0.87(6)$ & $1.44(7)$ \\
\hline$C(14)$ & $5.2(1)$ & $7.7(2)$ & $4.3(1)$ & $-2.4(1)$ & $-0.71(8)$ & $1.8(1)$ \\
\hline$C(15)$ & $4.56(9)$ & $4.15(9)$ & $2.47(7)$ & $0.60(6)$ & $0.76(6)$ & $2.04(7)$ \\
\hline$C(16)$ & $6.9(1)$ & $3.35(9)$ & $4.07(9)$ & $0.41(7)$ & $2.07(9)$ & $0.44(8)$ \\
\hline C (17) & $2.26(6)$ & $2.87(8)$ & $2.15(6)$ & $0.20(5)$ & $0.34(5)$ & $-0.24(5)$ \\
\hline C (18) & $3.72(8)$ & $3.11(8)$ & $2.69(8)$ & $-0.20(6)$ & $0.56(6)$ & $0.39(6)$ \\
\hline
\end{tabular}


Table s4. Bond lengths ( $\AA$ ) and angles (deg) with e.s.d.'s in parentheses.

\section{Bond Lengths}

$$
\begin{aligned}
& \mathrm{Ni}-\mathrm{O} \\
& \mathrm{Ni}-\mathrm{N}(2) \\
& \mathrm{O}-\mathrm{C}(1) \\
& \mathrm{C}(1)-\mathrm{C}(2) \\
& \mathrm{C}(2)-\mathrm{C}(3) \\
& \mathrm{C}(3)-\mathrm{C}(4) \\
& \mathrm{C}(3)-\mathrm{N}(4) \\
& \mathrm{N}(4)-\mathrm{C}(13) \\
& \mathrm{C}(13)-\mathrm{C}(14) \\
& \mathrm{N}(1)-\mathrm{C}(8) \\
& \mathrm{C}(8)-\mathrm{C}(9) \\
& \mathrm{C}(8)-\mathrm{C}(17) \\
& \mathrm{C}(9)-\mathrm{C}(18) \\
& \mathrm{C}(17)-\mathrm{N}(5)
\end{aligned}
$$

$$
\begin{aligned}
& \mathrm{Ni}-\mathrm{N}(1) \\
& \mathrm{Ni}-\mathrm{N}(3) \\
& \mathrm{C}(4)-\mathrm{C}(5) \\
& \mathrm{C}(5)-\mathrm{C}(6) \\
& \mathrm{C}(6)-\mathrm{C}(1) \\
& \mathrm{C}(6)-\mathrm{C}(7) \\
& \mathrm{C}(7)-\mathrm{N}(1) \\
& \mathrm{N}(4)-\mathrm{C}(15) \\
& \mathrm{C}(15)-\mathrm{C}(16) \\
& \mathrm{N}(2)-\mathrm{C}(9) \\
& \mathrm{N}(3)-\mathrm{C}(10) \\
& \mathrm{C}(10)-\mathrm{C}(11) \\
& \mathrm{C}(10)-\mathrm{C}(12) \\
& \mathrm{C}(18)-\mathrm{N}(6)
\end{aligned}
$$

\section{Bond Angles}

$$
\begin{aligned}
& \mathrm{O}-\mathrm{Ni}-\mathrm{N}(1) \\
& \mathrm{O}-\mathrm{Ni}-\mathrm{N}(2) \\
& \mathrm{O}-\mathrm{Ni}-\mathrm{N}(3)
\end{aligned}
$$$$
O-C(1)-C(6)
$$$$
O-C(1)-C(2)
$$$$
C(6)-C(1)-C(2)
$$$$
C(1)-C(2)-C(3)
$$$$
C(2)-C(3)-C(4)
$$$$
\mathrm{C}(2)-\mathrm{C}(3)-\mathrm{N}(4)
$$$$
\mathrm{C}(4)-\mathrm{C}(3)-\mathrm{N}(4)
$$$$
C(3)-C(4)-C(5)
$$$$
C(4)-C(5)-C(6)
$$$$
\mathrm{N}(1)-\mathrm{C}(8)-\mathrm{C}(9)
$$$$
\mathrm{N}(1)-\mathrm{C}(8)-\mathrm{C}(17)
$$$$
C(9)-C(8)-C(17)
$$$$
C(8)-C(17)-N(5)
$$$$
\mathrm{N}(3)-\mathrm{C}(10)-\mathrm{C}(11)
$$$$
\mathrm{N}(3)-\mathrm{C}(10)-\mathrm{C}(12)
$$$$
C(11)-C(10)-C(12)
$$$$
\mathrm{N}(4)-\mathrm{C}(13)-\mathrm{C}(14)
$$

$$
\begin{array}{r}
95.73(5) \\
176.57(5) \\
85.97(5)
\end{array}
$$

$\mathrm{Ni}-\mathrm{O}-\mathrm{C}(1)$
$123.06(12)$
$117.72(12)$
$119.22(12)$
$122.32(13)$
$117.73(13)$
$121.48(13)$
$120.79(13)$
$120.20(13)$
$122.71(13)$
$112.14(12)$
$123.20(12)$
$124.66(12)$
$178.30(15)$
$109.29(14)$
$110.73(13)$
$112.59(14)$
$112.27(14)$

$\mathrm{N}(1)-\mathrm{Ni}-\mathrm{N}(2)$

$\mathrm{N}(1)-\mathrm{Ni}-\mathrm{N}(3)$

$\mathrm{N}(2)-\mathrm{Ni}-\mathrm{N}(3)$

$127.79(9)$

$C(5)-C(6)-C(1)$

$C(5)-C(6)-C(7)$

$C(1)-C(6)-C(7)$

$\mathrm{C}(6)-\mathrm{C}(7)-\mathrm{N}(1)$

$\mathrm{Ni}-\mathrm{N}(1)-\mathrm{C}(7)$

$\mathrm{Ni}-\mathrm{N}(1)-\mathrm{C}(8)$

$\mathrm{C}(7)-\mathrm{N}(1)-\mathrm{C}(8)$

$\mathrm{Ni}-\mathrm{N}(2)-\mathrm{C}(9)$

$\mathrm{Ni}-\mathrm{N}(3)-\mathrm{C}(10)$

$\mathrm{N}(2)-\mathrm{C}(9)-\mathrm{C}(18)$

$\mathrm{N}(2)-\mathrm{C}(9)-\mathrm{C}(8)$

$C(8)-C(9)-C(18)$

$\mathrm{C}(9)-\mathrm{C}(18)-\mathrm{N}(6)$

$\mathrm{C}(3)-\mathrm{N}(4)-\mathrm{C}(13)$

$\mathrm{C}(3)-\mathrm{N}(4)-\mathrm{C}(15)$

$\mathrm{C}(13)-\mathrm{N}(4)-\mathrm{C}(15)$

$\mathrm{N}(4)-\mathrm{C}(15)-\mathrm{C}(16)$
$1.8664(11)$

$1.9259(12)$

$1.362(2)$

$1.4180(18)$

1.4261 (19)

1.4070 (19)

$1.3136(17)$

$1.457(2)$

$1.516(3)$

1.3306 (19)

1.4877 (18)

$1.507(2)$

$1.519(2)$

$1.144(2)$
$85.46(5)$
$175.83(5)$
$93.06(5)$

$117.55(12)$

$119.42(12)$

$123.02(12)$

$124.66(12)$

$125.38(9)$

112.08 (9)

122.53 (11)

$113.12(9)$

$116.62(10)$

$121.53(13)$

$117.14(12)$

121.32 (13)

179.51 (19)

$123.25(13)$

$121.20(12)$

$115.39(12)$

$112.72(13)$ 
Table s4. (Continued)

Shortest intra- and intermolecular Metal-Metal distances

$\mathrm{Ni} \ldots \mathrm{Ni}$

$6.6123(3)$

Ni...Ni(ii)

$$
\mathrm{Ni} \ldots \mathrm{Ni} \text { (iii) }
$$

Hydrogen bonds

\begin{tabular}{llllllr}
\hline $\mathrm{D}$ & $\mathrm{H}$ & $\mathrm{D}-\mathrm{H}$ & $\mathrm{A}$ & $\mathrm{H} \ldots \mathrm{A}$ & $\mathrm{D} \ldots \mathrm{A}$ & $\mathrm{D}-\mathrm{H} \ldots \mathrm{A}$ \\
\hline $\mathrm{N}(2)$ & $\mathrm{H}(2 \mathrm{~A})$ & 0.88 & $\mathrm{~N}(5 \mathrm{i})$ & 2.32 & $3.1131(17)$ & 150.6 \\
$\mathrm{~N}(3)$ & $\mathrm{H}(3 \mathrm{~A})$ & 0.92 & $\mathrm{O}$ & 2.29 & $2.5603(15)$ & 96.4 \\
& $\mathrm{H}(3 \mathrm{~B})$ & 0.92 & $\mathrm{~N}(6 \mathrm{i})$ & 2.64 & $3.266(2)$ & 125.8 \\
\hline
\end{tabular}

Symmetry operations:

$\begin{array}{rrrrr}1 & 2-X & 1-Y & 1-Z \\ i & 3 / 2-X & -1 / 2+Y & 1 / 2-Z \\ \text { ii } & 1 / 2+X & 1 / 2-Y & 1 / 2+Z \\ \text { iii } & -1 / 2+X & 1 / 2-Y & -1 / 2+Z\end{array}$


Table S5. Least-squares planes equations and deviations (̊) therefrom.

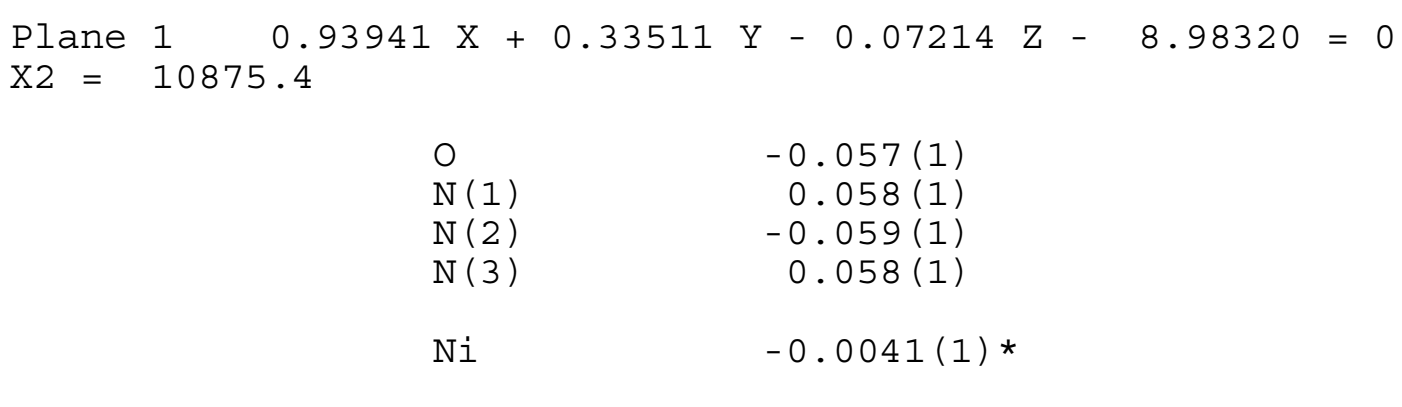

* denote atoms not included in planes calculations. 